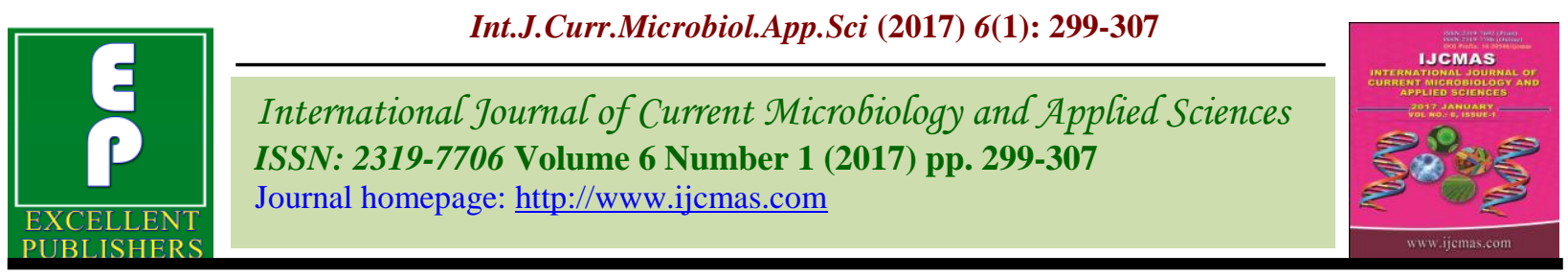

Original Research Article

http://dx.doi.org/10.20546/ijcmas.2017.601.036

\title{
Rapid Detection of Panton-Valentine Leukocidin (PVL) Gene from Staphylococcus aureus Clinical Isolates
}

\author{
Samia A. Girgis and Omnia M. Ahmed* \\ Department of Clinical Pathology, Faculty of Medicine, Ain Shams University, Egypt \\ *Corresponding author
}

A B S T R A C T

Infections caused by CA-MRSA have been associated with the presence of PantonValentine leukocidin (PVL) toxin which associated with increased disease severity, ranging from cutaneous infection requiring surgical drainage to severe chronic osteomyelitis and deadly necrotizing pneumonia. Panton-Valentine leukocidin (PVL) is a cytotoxin, one of the $\beta$-pore-forming toxins. PVL toxin targets cells of the human immune system like polymorphonuclear neutrophils (PMNs), monocytes, and macrophages

\begin{tabular}{|l|}
\hline Key w or d s \\
Panton-Valentine \\
Leukocidin (PVL) \\
Gene, \\
Staphylococcus \\
aureus.
\end{tabular}
forming pores on them that cause cytokine release and cell death by apoptosis or necrosis. The aim of this study is the rapid detection of PVL gene using real time PCR in S. aureus isolates recovered from community acquired $S$. aureus isolates from adult and pediatric infections, and to describe the prevalence of PVL gene positivity in community acquired. $S$. aureus strains. This study was conducted on one hundred and fifty clinical isolates of $S$. aureus isolates forming three groups (group one fifty community acquired isolates from adult infections, group two: fifty community acquired isolates from pediatric infections and group three:fifty hospital acquired isolates as a control group) isolated from different clinical specimens. All isolates were identified by conventional methods such as morphological identification, gram stain, and catalase test. Identification to species level by slide and tube coagulase, culture on DNase agar and mannitol salt agar. Then genotypic detection of PVL gene by real time polymerase chain reaction (PCR) was done. Regarding community acquired isolates from adults and pediatric infections $(88 \%)$ were positive for presence of PVL gene by real time PCR while all the control isolates were PVL gene negative, of these community acquired $S$. aureus isolates $(80 \%)$ were CA- MRSA while (20\%) were CA-MSSA. In the present study regarding different types of infection (100\%) of osteomyelitis were PVL gene positive but with no statistical significance. There was high statistical significance between skin and soft tissue infections and PVL gene positivity. There was also high statistical significance between skin soft tissue infection isolates and CA-MRSA. In conclusion, there is high percent of PVL gene positivity among CA-MRSA infections, so its rapid detection is recommended for early adjustment of antibiotic treatment.

\section{Introduction}

Staphylococcus aureus is a highly successful pathogen responsible for a variety of clinical problems ranging from folliculitis to endocarditis, osteomyelitis to pneumonia, and food intoxication to septic shock. A number of structural secreted virulence factors play a role in the pathogenesis of these various conditions and the evasion of 
host defenses. One of these exoproteins, Panton-Valentine leukocidin (PVL), is produced by several community-acquired methicillin-resistant $S$. aureus and methicillin-sensitive $S$. aureus (CA-MRSA and CA-MSSA, respectively) clones that are spreading throughout the world (Mandell and Wunderink, 2012).

Panton-Valentine leukocidin $(P V L)$ is a poreforming toxin which is composed of two protein components (LukF and LukS) that very efficiently disrupt the cell membrane of neutrophils. PVL has been associated with chronic or recurrent skin and soft tissue infections and with necrotizing pneumonia, which also affect immune competent persons (Masiuk et al., 2010). The adjunctive use of antibiotics that suppress toxin production, such as clindamycin, linezolid, and rifampin, and intravenous immunoglobulin is advocated for the treatment of severe and invasive infections caused by PVLproducing strains (Badiou, 2010). The prevalence of the PVL genes was less common among MSSA isolates than among MRSA isolates from infections and colonization in several studies (Davied and Daum, 2010).

Methicillin-resistant Staphylococcus aureus (MRSA) is a well-known public health problem that emerged shortly after the introduction of methicillin, nafcillin, and oxacillin antibiotics (Mangliner et al., 2008). In 2000, the CDC created a case definition for a CA-MRSA infection as any MRSA infection diagnosed for an outpatient or within 48 hours of hospitalization if the patient lacks the health care-associated MRSA risk factors (Davied and Daum, 2012).

The prevalence of CA-MRSA infections in the children population has progressively increased over the last decade (Teran et al.,
2012). CA-MRSA is known to cause predominantly skin and soft-tissue infections, but can also cause other severe community associated infections like myositis, pyomyositis, osteomyelitis and bacteremia (Rafee et al., 2012). These community-acquired MRSA (CA-MRSA) strains have epidemiological, clinical and microbiological features that distinguish them from the traditional hospital-based organisms Hospital Acquired MRSA (HAMRSA) (Pantost and venditti, 2009).

Methicillin resistance is conferred by the mecA gene, which is located in the staphylococcal cassette chromosome mec (SCC mec). The SCC mec elements are different in HA-MRSA and CA-MRSA strains. HA-MRSA strains typically contain the larger type I, II, or III elements, whereas CA-MRSA strains contain the smaller type IV or V elements (Frtiz et al., 2010). CAMRSA strains differ from their HA-MRSA counterparts by usually being susceptible to clindamycin and other non-B-lactam antibiotics. They also typically carry the 2 genes encoding Panton-Valentine leukocidin (PVL) (Davied et al., 2008).

Therefore, detection of PVL gene helps in treatment and diagnosis of community acquired MRSA. One of the methods for detection of PVL gene is real time polymerase chain reaction (PCR), Real-time PCR is significantly faster than other detection methods. The combination of excellent sensitivity and specificity, low contamination risk, ease of performance and speed has made real-time PCR technology appealing to the clinical microbiology laboratory (Jukes et al., 2010).

The aim of this study isthe rapid detection of $P V L$ gene using real time PCR in $S$. aureus isolates recovered from community acquired 
S. aureus isolates from adult and pediatric infections, and to describe the prevalence of PVL gene positivity in community acquired S. aureus strains.

\section{Materials and Methods}

This study was conducted on one hundred and fifty S. aureus clinical isolates that were collected from various clinical specimens, submitted for routine culture and susceptibility testing to the Main Microbiology Laboratory, Ain Shams University Hospitals, Cairo, Egypt. The isolates were collected as three groups (group one fifty community acquired isolates from adult infections, group two fifty community acquired isolates from pediatric infections and group three fifty hospital acquired isolates as control).

Isolates for adult and pediatric groups were collected as clinical isolates of $S$. aureus from specimens of out-patient clinic and recently hospitalized patients within 48 hours of hospitalization.

Isolates for Control group were collected as clinical isolates from hospitalized patients having one or more of the following health care-associated $S$. aureus risk factor: Hemodialysis, Surgery, Residence in a longterm care facility or hospitalization during the previous year, and Presence of an indwelling catheter or a percutaneous device at the time of culture, representing hospital acquired $S$. aureus infections.

Data was collected from patients about risk factors, antibiotics intake, associated medical diseases and relation to medical staff.

All isolates were identified using conventional methods as Gram stain and catalase. Then, the isolates were identified to the species level by slide and tube coagulase test, subculture on DNAse agar, and mannitol salt agar. Antimicrobial susceptibility testing was carried out by the disk diffusion method according to CLSI guidelines using the interpretative criteria for cefoxitin, clindamycin, linezolid and rifampicin.

The isolates were stored in aliquots containing tryptic soya broth (Oxoid, UK.) at $-70^{\circ} \mathrm{C}$ till used for Real-time PCR for detection of $P V L$ gene.

\section{Polymerase chain reaction}

Real time Polymerase chain reaction (PCR) (Rotor gene 5plex) was performed for detection of PVL genein the 150 isolates of adult, pediatric and control groups. Bacterial DNA was extracted using DNA extraction kit; QIA amp DNA Mini Kit supplied by (Qiagen, USA). The reaction mixture included Quanti Tect SYBR Green PCR Master Mix (Qiagen, USA), Real time PCR was done using the following primers for $P V L$ gene:

*PVL Forward: 5'ATG TCC TTT CAC TTT AAT TTC ATG AGT TTT 3'.

**PVL Reversed:5 CAT GCT ACT AGA AGA ACA ACA CAC TAT GG 3' (Shallcross, et al., 2010).

The PCR conditions were with initial denaturation step at $95^{\circ} \mathrm{C}$ for $10 \mathrm{~min}$ and 38 cycles of amplification consisting of: denaturation at $95^{\circ} \mathrm{C}$ for $15 \mathrm{~s}$., annealing at $60^{\circ} \mathrm{C}$ for $30 \mathrm{~s}$, extension at $72^{\circ} \mathrm{C}$ for $30 \mathrm{~s}$. Detection of the PCR amplified product was done by SYBR Green (Qiagen, USA).

\section{Results and Discussion}

Regarding detection of PVL gene by PCR, PVL gene was positive in $42(84 \%)$ of community acquired isolates in adult group, $46(92 \%)$ in pediatric group while all the 
hospital acquired isolates (control group) were PVL gene negative, with a highly statistical significance between the two groups ( $\mathrm{p}$ value <0.001) (table 1).

Out of 50 community acquired $S$. aureus isolates $46(92 \%)$ in adult group and 34 $(68 \%)$ in pediatric group were CA- MRSA. While $4(8 \%)$ in adult group and $16(32 \%)$ in pediatric group were CA-MSSA. Whereas in the control group out of 50 hospital acquired isolates $47(94 \%)$ were HA-MRSA and 3 (6\%) were HA-MSSA.

Out of 46 CA- MRSA 38 (76\%) were PVL gene positive in adult group while out of 34 CA- MRSA 31 (91\%) were PVL gene positive in pediatric group. There is higher percentage of PVL gene positivity among cases of CA-MRSA but there was no statistical significance between CA-MRSA and CA-MSSA as regards PVL positivity ( $\mathrm{P}$ value $>0.05$ ).

In the adult group, Out of $50 \mathrm{~S}$. aureus isolates $32(64 \%)$ caused infections that required hospitalization and the isolates were recovered from specimens in the first 48 hours of hospitalization and $18(36 \%)$ were from outpatient clinic. There is a higher percentage of PVL gene positivity among recently hospitalized patients $28(87.5 \%)$ out of 32 compared to $14(77.77 \%)$ out of 18 of outpatients and it is statistically not significant $(P$ value $=0.3)$. While in pediatric group, Out of 50S. aureus isolates $36(72 \%)$ were from recently hospitalized patients with $35(97.2 \%)$ of isolates were PVL gene positive, While 14 of 50 (28\%) of isolates were from outpatients with $11(78.6 \%)$ of isolates were PVL gene positive. There is a higher percentage of PVL gene positivity among recently hospitalized patients $(97.2 \%)$ compared to $(78.6 \%)$ of outpatients and it is statistically not significant $(P$ value $=0.06)$.
Out of $50 \mathrm{~S}$. aureus isolates for each adult and pediatric groups 14 (28\%), 20 (40\%) were from patients taking antibiotics, 4 (8\%) from patients with history of contact to relative medical staff, and 13 (26\%), 14 (28\%) of patients having associated medical diseases in adults and pediatric groups respectively. As regard PVL gene positivity there was no statistically significant association with any of these risk factors in either of the groups $(\mathrm{p}>0.05)$.

In the adult group, The fifty $\mathrm{S}$. aureus isolates were collected from different types of infection, out of 50 isolates $28(56 \%)$ were from skin and soft tissue followed by pneumonia 9 (18\%) followed by UTI 6 $(12 \%)$ then bacteremia $5(10 \%)$ and osteomyelitis $2(4 \%)$. There were $23(46 \%)$, 7 (14\%), 6 (12\%), 4(8\%), 2(4\%) PVL gene positive $S$. aureus isolates respectively. It was found that $100 \%$ of osteomyelitis were PVL gene positive. On comparing the results of PVL gene according to different types of infections, there is high statistically significant association between soft tissue infections, UTI and PVL gene presence $(\mathrm{P}<$ 0.01 ) and there is a border line significance for pneumonia ( $\mathrm{P}$ value $=0.05$ ). There was also high statistical significance between CA-MRSA and skin and soft tissue infection cases. While in the pediatric group, out of 50 CA S. aureus isolates 22 (44\%) were from skin and soft tissue followed by osteomyelitis 12 (24\%), and the lowest percentage was from bacteremia $5(10 \%)$ and UTI $5(10 \%)$ then pneumonia $3(6 \%)$ and other infections $3(6 \%)$.It was found that $100 \%$ of isolates from this infections were PVL gene positive. On comparing the results of PVL gene positivity according to different type of infections, there was no statistical significance $(\mathrm{P}>0.05)$.In the control group, out of $50 \mathrm{HA} S$. aureus isolates $23(46 \%)$ were from skin and soft tissue followed by bacteremia $13(26 \%)$, and the lowest 
percentage was from pneumonia 12(24\%) and UTI $0(0 \%)$ then osteomyelitis $0(0 \%)$ and other infections $2(4 \%)$.

In the adult group, out of $50 \mathrm{~S}$. aureus isolates 20 (40\%), 19 (38\%), 17(34\%) were sensitive to clindamycin, linezolid and rifampicin respectively, whereas $30(60 \%)$, $31(62 \%), 33(66 \%)$ were resistance to clindamycin, linezolid and rifampicin respectively. For pediatric group, out of $50 \mathrm{~S}$. aureus isolates 34 (85\%), 26 (52\%) were sensitive to clindamycin, and rifampicin respectively, whereas 16 (15\%), 24 (48\%) were resistance to clindamycin, and rifampicin respectively. All S. aureus isolates from pediatric group were sensitive to Linezolid antibiotic. There was no statistical significance between antimicrobial susceptibility and PVL gene positivity. While in the control group,out of $50 \mathrm{~S}$. aureus isolates 21 (42\%), 36 (72\%), 9(18\%) were sensitive to clindamycin, linezolid and rifampicin respectively, whereas 29 (58\%), $14(28 \%), 41(82 \%)$ were resistance to clindamycin, linezolid and rifampicin respectively.Only Linezolid had statistically higher sensitivity in the hospital acquired control group $36(72 \%)$ than the community acquired adult group $19(38 \%)(\mathrm{p}<0.01)$. The percentage of PVL gene positivity is higher among resistant strains of $S$. aureus to Clindamycin (46\%), Linezolid (46.8\%) andRifampicin (54\%). A statistically significant association was found between PVL gene positivity and Linezolid resistance $(\mathrm{p}<0.05)$ only.

Staphylococcus aureus causes a range of infections, from mild skin infections to septicemia. Shortly after the introduction of methicillin in the early 1960s, methicillinresistant S. aureus (MRSA) emerged and now causes serious nosocomial infections worldwide. Despite its spread in hospitals and nursing homes, MRSA has not disseminated in the wider community until recently. Such infections not acquired at a healthcare setting or institution is considered community associated MRSA (CA-MRSA). The discovery of Panton-Valentine leukocidin (PVL), encoded by lukF-PV and lukS-PV genes, was reported in the 1930s, and PVL has been associated with $1.6 \%$ of $S$. aureus strains in England and Wales in 2002. MRSA strains producing PVL toxin have been associated with an increase in severe skin infections and a new syndrome of community-associated necrotizing pneumonia in children. The PVL genes are carried on the phage SLT, but PVL has not been identified with epidemic or endemic hospital-acquired MRSA strains (Rayan et $a l ., 2005)$. The present study aimed to detect the PVL gene in community acquired $\mathrm{S}$. aureus as one of the virulence factors of community acquired S. aureus and also in hospital acquired S. aureus (control) using real-time PCR as one of the rapid and accurate methods.

This study was conducted on one hundred and fifty S. aureus clinical isolates that were collected from various clinical specimens. The isolates were collected as three groups (group one fifty community acquired isolates from adult infections, group two fifty community acquired isolates from pediatric infections and group three fifty hospital acquired isolates as control). All isolates were identified using conventional methods as Gram stain and catalase. Then, the isolates were identified to the species level by slide and tube coagulase test, subculture on DNAse agar, and mannitol salt agar.Antimicrobial susceptibility testing was carried out by the disk diffusion method according to CLSI guidelines using the interpretative criteria for cefoxitin, clindamycin, linezolid and rifampicin. Realtime PCR was made for detection of $P V L$ gene. 
In the current study, PVL gene was positive in $42(84 \%)$ of community acquired isolates in adult group, $46(92 \%)$ in pediatric group while all the hospital acquired isolates (control group) were PVL gene negative, Similarly Baran et al., 2010 conducteda study on thirty strains that were phenotypically identified as MRSA and after assessing the risk factors, 28 (93.3\%) of them were classified as health-care associated (HCA) and $2(6.7 \%)$ of them as community-acquired (CA). Panton-Valentin leucocidin (PVL) gene positivity was detected in only CA-MRSA isolates (2/2; $100 \%)$.Panton-Valentin leucocidin (PVL) genes have been identified as a stable marker of CA-MRSA strains worldwide (Morrison et al., 2006).

On the other hand, WD et al., 2010 conducted a study on $1104 \mathrm{~S}$. aureus isolates community acquired and hospital acquired. $31.8 \%$ (351) were community-acquired $S$. aureus. Panton-Valentine Leukocidin (PVL)-positive community-acquired $S$. aureus strains were $35.3 \%$ only. Also Gülmez et al., 2012 conducted a study to detect SCCmec types and PVL gene in S.aureus strains isolated from various infections. They found that Ten out of 446 (2.2\%) were PVL positive strains that were all methicillin susceptible S.aureus (MSSA).Muttaiyah et al., 2010 stated that in Auckland, PVL-positive MSSA was strongly associated with the diagnosis of SSTI; $48 \%$ of all MSSA isolates associated with SSTI were lukSF-PV positive. The reported incidence of PVL-positive MSSA SSTI in the literature ranges from $6.8 \%$ in a teaching hospital in Michigan to $93 \%$ in a New York prison endemic with the PVL-positive USA300 MSSA strain (Muttaiyah et al., 2010).

In the current study out of $100 \mathrm{~S}$. aureus isolates $89(89 \%)$ are MRSA, followed by MSSA which form $11(11 \%)$ of the total study isolates. In a similar study done in Sudan by Kheder et al., 2012, Out of 35 strains of $S$. aureus isolated from surgical samples, $25(71.4 \%)$ were found to be MRSA. Levels reported elsewhere differed substantially ranging from $22 \%-65 \%$. However, there are evidences that the prevalence of MRSA has increased worldwide (Kheder et al., 2012).On the other hand a study made by Egyir et al., 2014 in Ghana on 903 staphylococci collected, 308 (34\%) were identified as S. aureus and 595 $(66 \%)$ as coagulase negative staphylococci. MRSA and MSSA isolates formed nearly (3\%), (97\%) respectively. The prevalence of MRSA (3\%) was lower than those reported in other African countries such as Nigeria (20\%), Algeria (45\%) and in a multicenter study $(15 \%)$ involving five major African towns. Egyir et al., 2014 attributed the low MRSA frequency reported in their study to the low consumption of antimicrobial agents such as fluoroquinolones and third generation cephalosporins in Ghana, because they are expensive and are usually prescribed for acute infections. Usage of the aforementioned antimicrobial agents has been shown to correlate with an increase in MRSA prevalence (Egyir et al., 2014). In another western country WD et al., 2010 found that CA-MRSA accounted for 4\% (14) of $S$. aureus isolates.

Also Shallcross et al., 2010 conducted a study on 390 isolates in England, they found that Methicillin-resistant S. aureus with PVL was rare $(0.8 \%$ of all isolates) but PVL with methicillin-sensitive S. aureus was common (9.0\% of all specimens). Also Gülmez et al., 2012 found MRSA to be 97 (34\%) out of 285 S. aureus isolates. The observed MRSA prevalence among clinical isolates in Ghana is similar to those reported in European countries with low MRSA prevalence, such as the Scandinavian countries and The Netherlands (Egyir et al., 2014). 
Table.1 Comparison between case group and control group regarding PVL gene presence

\begin{tabular}{|l|l|l|l|l|}
\hline $\begin{array}{l}\text { Staphylococcus } \\
\text { aureus isolates }\end{array}$ & $\begin{array}{l}\text { Community } \\
\text { acquired } \\
\text { group }\end{array}$ & $\begin{array}{l}\text { Hospital } \\
\text { acquired } \\
\text { Control group }\end{array}$ & $\mathbf{X}^{\mathbf{2}}$ & p value \\
\hline PVL positive & $88(88 \%)$ & $0(0 \%)$ & 72.414 & $<0.001$ \\
\hline PVL negative & $12(12 \%)$ & $50(100 \%)$ & & \\
\hline
\end{tabular}

In the current study, there is high statistically significant association between soft tissue infections, UTI and PVL gene presence $(\mathrm{P}<$ 0.01 ) and there is a border line significance for pneumonia ( $\mathrm{P}$ value $=0.05$ ). Simiraly, WD et al., 2010found that Panton-Valentine Leukocidin (PVL)-positive communityacquired $S$. aureus strains were more commonly associated with skin abscesses than other SSTIs $(29.4 \%$ versus $5.9 \%, P<$ 0.01).Also Shallcross et al., 2010found that $9.7 \%$ of clinical isolates and $20.8 \%$ of skin and soft tissue specimens contained the genes for PVL. Also Gülmez et al., 2012 isolated 7 PVL positive strains out of 446 $(1.5 \%)$ causing skin and soft tissue infections. They stated that CA-MRSA strains might harbour Panton-Valentine leukocidin (PVL), an important virulence factor in skin and soft tissue infections. They added that Strains carrying PVL has the ability to penetrate undamaged skin and cause more severe infections.

In the current study, antimicrobial resistance rates of CA-MRSA and HCA-MRSA isolates were found as follows, respectively $46 \%$ and $58 \%$ for clindamycin, $57 \%$ and $82 \%$ for rifampicin and, $31 \%$ and $72 \%$ for linezolid. All S. aureus isolates in pediatric group were sensitive to Linezolid antibiotic. Also Baron et al., 2010reportedthe antimicrobial resistance rates of HCAMRSA and CA-MRSA isolates were found as follows; $89.3 \%$ and $0 \%$ for rifampin, $89.3 \%$ and $50 \%$ for ciprofloxacin, $89.3 \%$ and $0 \%$ for gentamicin, $50 \%$ and $50 \%$ for erythromycin, $28.6 \%$ and $0 \%$ for clindamycin, whereas all of the isolates were susceptible to vancomycin, linezolid and trimethoprim-sulfamethoxazole. WD et al., 2010 found that among 14 CA-MRSA and 120 MSSA isolates tested, $100 \%$ and $91.7 \%$ were multidrug resistant, respectively. The high antimicrobial resistance rates could be attributed to the high consumption of antimicrobial agents.

In conclusion there is a high percent of PVL gene positivity among CA-MRSA infections, so its rapid detection is recommended for early adjustment of antibiotic treatment. As the adjunctive use of antibiotics that suppress toxin production, such as clindamycin, linezolid, and rifampin, and intravenous immunoglobulin is advocated for the treatment of severe and invasive infections caused by PVL-producing strains. More studies are required for implementing cheaper methods for detection of PVL toxin directly from specimens such as specific enzymelinked immunosorbent assay (ELISA) or immunochromatographic test (ICT) methods, a step for routine work up for PVL toxin detection in $S$. aureus clinical isolates.

\section{References}

Badiou, C., Dumitrescu, O., George, N., Forbes, A., et al. 2010. Rapid detection of Staphylococcus aureus PantonValentine leukocidin in clinical specimens by enzyme-linked immunosorbent assay and immunochromatographic tests. J. Clin. Microbiol., 48(4): 1384-1390. 
Baran, C.B., Mutlu,

D., Baysan, B.O., Günseren, F., Ergani, A., Oğünç, D., Colak, D. 2010. Investigation of Panton-Valentine leukocidin gene, SCCmec gene cassette types and genotypes of methicillin-resistant Staphylococcus aureus strains isolated from outpatients. Mikrobiyol. Bul., 44(4): 533-45.

David, M.Z., Glikman, D., Crawford, S.E., Peng, J., King, K. J., Hostetler, M.A., et al. 2008. What is community-associated methicillin-resistant Staphylococcus aureus? J. Infect. Dis., 197(9): 12351243.

David, Z.M. and Daun, S.R. 2010. Community-Associated MethicillinResistant Staphylococcus aureus: Epidemiology and Clinical Consequences of an Emerging Epidemic. Clin. Microbiol. Rev., 23(3): 616-687.

Egyir, B., Guardabassi, L., Sørum, M., Nielsen, S.S., Kolekang, A., et al. 2014. Molecular Epidemiology and Antimicrobial Susceptibility of Clinical Staphylococcus aureus from Healthcare Institutions in Ghana. PLoS ONE, 9(2): e89716.

Fritz, S.A., Epplin, E.K., Garbutt, J. and Storch, G.A. 2010. Skin infection in children colonized with community associated methicillin resistant staphylococcus aureus. NIH Public Access J. Infect., 59(6): 394-401.

Gülmez, D., Sancak, B., Ercis, S., Karakaya, J., Hasçelik, G. 2012. Investigation of SCCmec types and Panton-Valentine leukocidin in community-acquired and nosocomial Staphylococcus aureus strains: comparing skin and soft tissue infections to the other infections. Mikrobiyol. Bul., 46(3): 341-51.

Kheder, S.I., Ali, A.N., and Fathelrahman, A.I. 2012. Prevalence and Antimicrobial Susceptibility Pattern of Methicillin Resistance Staphylococcus in a Sudanese Surgical Ward. Pharmacol. Pharm., 3: 103-108.

MandellL, A. and Wunderink, R. 2012. Methicillin-Resistant Staphylococcus aureus and Community-Acquired Pneumonia: An Evolving Relationship. Clin. Infect. Dis., (8):1134-113

Morrison, M.A., Hageman, J.C., and Klevens, R.M. 2006. Case definition for community-associated methicillinresistant Staphylococcus aureus. $J$. Hospital Infect., 62(2): 241.

Muttaiyah, S., Coombs, G., Pandey, S., Reed, P., Ritchie, S., Lennon, D., and S. Roberts. 2010. Incidence, Risk Factors, and Outcomes of Panton-Valentine Leukocidin-Positive MethicillinSusceptible Staphylococcus aureus Infections in Auckland, New Zealand. J. Clin. Microbiol., 48(10): 3470-3474.

Pantosti, A. and Venditti, M. 2009. What is MRSA?. European respiratory J., 34(5): 1190-1196.

Rafee, Y., Abdel-Haq, N., Asmar, B., Salimnia, T., Pharm, C. V., Rybak Pharm, M.J. \& Amjad, M. 2012. Increased prevalence of methicillinresistant Staphylococcus aureus nasal colonization in household contacts of children with community acquired disease. BMC infectious dis., 12(1): 45

Ryan, R., McDonald, Nick, A., Antonishyn, Toni Hansen, Laelie, A., Snook, Evelyn Nagle, Michael, R., Mulvey, Paul N. Levett, and Greg B. Horsman. 2005. Development of a Triplex Real-Time PCR Assay for Detection of PantonValentine Leukocidin Toxin Genes in Clinical Isolates of MethicillinResistant Staphylococcus aureus. $J$. Clin. Microbiol., 43 (12): 6147-6149.

Shallcross, L.J., Williams, K., Hopkins, S., Aldridge, R.W., Johnson, A.M. and Hayward, A.C. 2010. Panton-Valentine leukocidin associated staphylococcal disease: a cross-sectional study at a 
London hospital, England. Clin. L., Shen. 2010. Epidemiology and Microbiol. Infect., 16: 1644-1648

Teran, C.G., Sura, S., Thant Lin, T.M., Medows, M., et al. 2012. Current role of community-acquired methicillinresistant Staphylococcus aureus among children with skin and soft tissue infections. Pediatric reports, 4(1): e5. molecular characteristics of communityassociated methicillin-resistant and methicillin-susceptible Staphylococcus aureus from skin/soft tissue infections in a children's hospital in Beijing, China. Diagn. Microbiol. Infect. Dis., 67(1): 1-8.

Wu, D., Wang, Q., Yang, Y., Geng, W., Wang, Q., Yu, S., Yao, K., Yuan,

\section{How to cite this article:}

Samia A. Girgis and Omnia M. Ahmed. 2017. Rapid Detection of Panton-Valentine Leukocidin (PVL) Gene from Staphylococcus aureus Clinical Isolates. Int.J.Curr.Microbiol.App.Sci. 6(1): 299-307. doi: http://dx.doi.org/10.20546/ijcmas.2017.601.036 